\title{
The canonical coloring graph of trees and cycles*
}

\author{
Ruth Haas ${ }^{\dagger}$ \\ Smith College, Northampton, MA 01063, USA
}

Received 1 August 2010, accepted 13 October 2011, published online 20 January 2012

\begin{abstract}
For a graph $G$ and an ordering of the vertices $\pi$, the set of canonical $k$-colorings of $G$ under $\pi$ is the set of non-isomorphic proper $k$-colorings of $G$ that are lexicographically least under $\pi$. The canonical coloring graph $\operatorname{Can}_{k}^{\pi}(G)$ is the graph with vertex set the canonical colorings of $G$ and two vertices are adjacent if the colorings differ in exactly one place. This is a natural variation of the color graph $\mathcal{C}_{k}(G)$ where all colorings are considered. We show that every graph has a canonical coloring graph which is disconnected; that trees have canonical coloring graphs that are Hamiltonian; and cycles have canonical coloring graphs that are connected.
\end{abstract}

Keywords: Graph coloring, Canonical coloring

Math. Subj. Class.: 05C15

\section{Introduction}

For a positive integer $k$ and a graph $G$, the $k$-coloring graph of $G, \mathcal{C}_{k}(G)$, is the graph whose vertex set is the set of proper $k$ vertex colorings of $G$. Two $k$ colorings are joined by an edge if they differ in color on just one vertex of $G$. The basic question of whether $\mathcal{C}_{k}(G)$ is connected has recieved significant attention, see for example [1, 5, 6, 8]. In theoretical physics $\mathcal{C}_{k}(G)$ appears in the Glauber dynamics of an anti-ferromagnetic Potts model at zero temperature. In Glauber dynamics, the state space of the Markov chain is the set of all $k$-colorings of a graph. Markov processes also give a method to approximate the number of $k$-colorings of $G$. If $\mathcal{C}_{k}(G)$ is connected, the probabilities of moving between colorings in $\mathcal{C}_{k}(G)$ can be assigned so that the stationary distribution is uniform on all proper colorings. If the process converges fast enough (in polynomial time) then repeated sampling can be used to obtain a reasonable approximation of the total number of proper colorings. There is an extensive literature on when there is rapid mixing of $\mathcal{C}_{k}(G)$ see for example $[5,6,7,8]$.

\footnotetext{
* In memory of Michael Albertson, who provided primary colors in my graph theory toolbox.

${ }^{\dagger}$ This research was partially supported by NSF grant DMS-0721661.

E-mail address: rhaas@ smith.edu (Ruth Haas)
} 


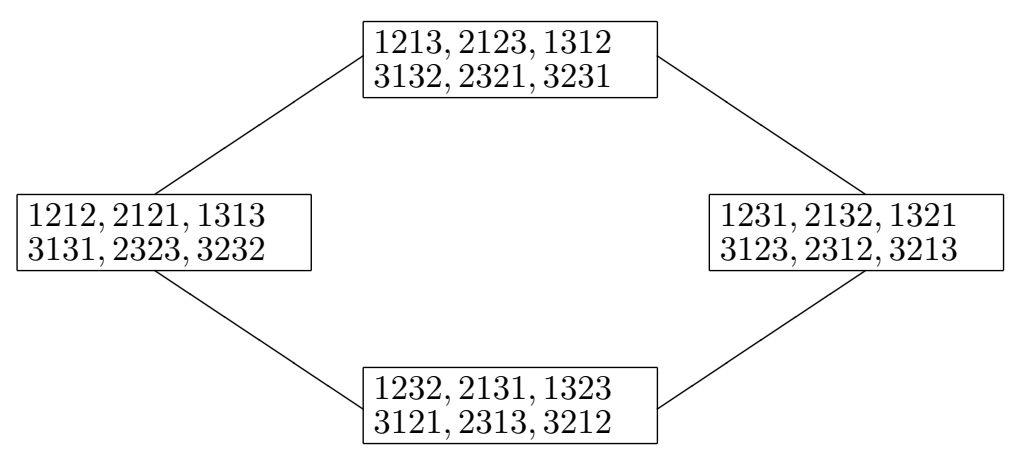

Figure 1: $I_{3}\left(P_{4}\right)$

Recently, several groups of graph theorists have given conditions which ensure the connectivity of $\mathcal{C}_{k}$ for classes of graphs. Moreover, they have shown that there is no such condition that depends only on the chromatic number $\chi(G)$.

Proposition 1.1 (Cereceda, van den Heuvel, and Johnson [1]). There is no function $F(\chi)$, so that for all graphs $G$ and integers $k>F(\chi(G)), \mathcal{C}_{k}(G)$ is connected.

Recall the coloring number, $\operatorname{col}(G)$, is the smallest integer $t$ for which there exists an ordering of the vertices $v_{1}, \ldots v_{n}$, such that for all $i$ the degree of $v_{i}$ in the induced graph on $v_{1}, \ldots v_{i}$ is less than $t$. That is, there exists an ordering of the vertices so that $G$ can be greedily colored using $t$ colors.

Theorem 1.2 (Dyer, Flaxman, Frieze, Vigoda [3]). For any graph $G$ and integer $k \geq$ $\operatorname{col}(G)+1, \mathcal{C}_{k}(G)$ is connected.

Beyond whether $\mathcal{C}_{k}(G)$ is connected, MacGillivray and Choo have shown that for all graphs $G, \mathcal{C}_{k}(G)$ is Hamiltonian for sufficiently large $k$.

Theorem 1.3 (Choo and MacGillivray [2]). If $k \geq \operatorname{col}(G)+2$, then $\mathcal{C}_{k}(G)$ is Hamiltonian.

In this paper we consider only non-isomorphic colorings of a graph $G$ and give some conditions for when the graph of such colorings is connected and Hamiltonian. Section 2 provides definitions and basic results. Sections 3 and 4 give results for trees and cycles respectively.

\section{The canonical coloring graph}

Two colorings of $G$ are isomorphic if one results from permuting the names of the colors only (i.e, we do not consider automorphisms of the graph). They are non-isomorphic otherwise. It is natural to consider the variation of the coloring graph where the vertices correspond to isomorphism classes of colorings of $G$. For simplicity, we will refer to each vertex as a coloring of $G$ (rather than an isomorphism class of colorings). There are several ways to define the edge set. This paper will mainly look at the canonical coloring graph defined below. Another possibility is the isomorphic coloring graph, $I_{k}(G)$, which is defined to be the graph with an edge between two colorings $c, d$ if some representative of $c$ 
differs at exactly one vertex from some representative of $d$. Figure 1 shows $I_{3}\left(P_{4}\right)$, where $P_{4}$ is the path on 4 vertices.

Proposition 2.1. If $\mathcal{C}_{k}(G)$ is connected then so is $I_{k}(G)$.

Proof. There is natural graph homomorphism from $\mathcal{C}_{k}(G)$ to a multigraph which is $I_{k}(G)$ with the addition of loops at each vertex.

On the other hand, $I_{k}(G)$ may be connected when $\mathcal{C}_{k}(G)$ is not. For example, $I_{3}\left(C_{5}\right)$ is also $C_{5}$ while $\mathcal{C}_{3}\left(C_{5}\right)$ is two disjoint 15 cycles.

For a graph $G$ and an ordering of the vertices $\pi$, the set of canonical $k$-colorings of $G$ under $\pi$ is the set of non-isomorphic proper $k$-colorings of $G$ that are lexicographically least under $\pi$. The canonical coloring graph $\operatorname{Can}_{k}^{\pi}(G)$ is the graph whose vertices are the canonical colorings of $G$ where two vertices are adjacent if the colorings differ in exactly one place. The order $\pi$ of vertices of $G$ can result in different $\operatorname{Can}_{k}^{\pi}(G)$. For example, Figure 2 shows $\operatorname{Can}_{3}^{\pi_{i}}\left(P_{4}\right)$ for three different orderings of the vertices. In each case the path is formed by edges $\{(1,2),(2,3),(3,4)\}$. If the vertices are colored in the same order as the path, $\pi_{1}=(1,2,3,4)$, the canonical coloring graph $\operatorname{Can}_{3}^{\pi_{1}}\left(P_{4}\right)$ is a path (Figure 2a). For $\pi_{2}=(1,3,2,4)$, canonical coloring graph $\operatorname{Can}_{3}^{\pi_{2}}\left(P_{4}\right)$ is a pair of disjoint edges (Figure 2b). For $\pi_{3}=(3,2,1,4)$, canonical coloring graph $\operatorname{Can}_{3}^{\pi_{2}}\left(P_{4}\right)$ is a cycle (Figure 2c). Thus properties of $\operatorname{Can}_{k}^{\pi}(G)$ may depend on the order $\pi$. Indeed, whenever $G$ is not a complete graph there exists an order for which $\operatorname{Can}_{k}^{\pi}(G)$ is disconnected.

Theorem 2.2. Let $G \neq K_{n}$ be a connected graph and $k \geq \chi(G)+1$. Then there exists an order $\pi$ such that $\operatorname{Can}_{k}^{\pi}(G)$ is disconnected.

Proof. Observe that if $G$ is connected on $n>2$ vertices and not complete then it contains an induced $P_{3}$. Let $\pi$ be an ordering of the vertices $(a, b, c, \ldots)$ such that $(a, c),(b, c) \in$ $E(G)$ and $(a, b) \notin E(G)$. Now every canonical coloring of $G$ must begin either $112 \ldots$ or $123 \ldots$. . Every coloring of the first kind differs in (at least) two places from every coloring of the second kind, thus as long as there is at least one coloring of each kind, the graph $\operatorname{Can}_{k}^{\pi}(G)$ is disconnected. Since there is a canonical proper coloring with $\chi(G)$ colors, at least one of coloring will be obtained with $\chi(G)$ colors. A proper coloring of the first kind is obtained by taking a proper coloring of $G$ with exactly $\chi(G)$ colors, changing the color on vertices $a$ and $b$ to the $(\chi+1)$ color and recoloring to get the canonical coloring isomorphic to this. A proper coloring of the second kind is obtained by taking a proper coloring of $G$ with exactly $\chi(G)$ colors, changing only the color on vertex $b$ to the $(\chi+1)$ color and recoloring for the canonical coloring isomorphic to this.

\section{Trees}

In this section we give conditions so that the canonical coloring graph of a tree has a Hamilton cycle.

Theorem 3.1. For $T$ a tree with $n \geq 4$ vertices, and $k \geq 3$ colors, there is an order $\pi$ of the vertices such that $\operatorname{Can}_{k}^{\pi}(T)$ has a Hamilton cycle.

Proof. The proof is in three parts. We first show the result for stars, then for trees using at least 4 colors, and finally for trees using exactly three colors. 


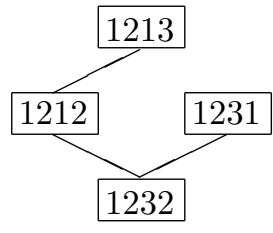

(a)

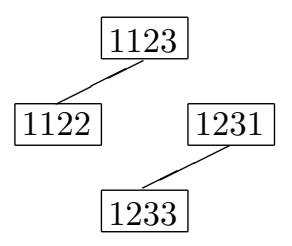

(b)

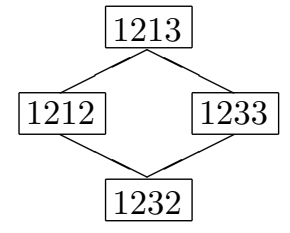

(c)

Figure 2: $P_{4}$ and $\operatorname{Can}_{3}^{\pi}\left(P_{4}\right)$ for three different orderings of the vertices of $P_{4}$. The colors are listed in the order given by the permutation.

Part I: Stars. Let $S_{n}$ be the star with $n \geq 4$ vertices and $k \geq 3$ colors, let $\pi$ be an ordering of the vertices such that the root is first.

We first address the case where $k=3$. It is straightforward to check if $n \leq 3$ $\operatorname{Can}_{3}^{\pi}\left(S_{n}\right)$ has a Hamilton path (though not a cycle). Assume now there is a Hamilton path on $\operatorname{Can}_{3}^{\pi}\left(S_{n-1}\right)$, specifically colorings $c_{1}, c_{2}, \ldots, c_{N}$. Every coloring of $S_{n}$ is obtained by taking a coloring $c_{i}$ of $S_{n-1}$ and choosing either color 2 or 3 for the $n$th vertex. Now $\operatorname{Can}_{3}^{\pi}\left(S_{n}\right)=\operatorname{Can}_{3}^{\pi}\left(S_{n-1}\right) \otimes K_{2}$, the Cartesian product. Hence $\operatorname{Can}_{3}^{\pi}\left(S_{n}\right)$ has a Hamilton cycle if $\operatorname{Can}_{3}^{\pi}\left(S_{n-1}\right)$ has a Hamilton path.

For $k>3$, if $n=1,2,3, \operatorname{Can}_{k}^{\pi}\left(S_{n}\right)=\operatorname{Can}_{3}^{\pi}\left(S_{n}\right)$ has a Hamilton path. A Hamilton cycle on $\operatorname{Can}_{k}^{\pi}\left(S_{4}\right)$ is given by $1223,1222,1232,1234,1233$.

Assume there is a Hamilton cycle on $\operatorname{Can}_{k}^{\pi}\left(S_{n-1}\right)$, specifically colorings $c_{1}, c_{2}, \ldots, c_{N}$. Choose $c_{N}, c_{1}$ so that they both use at least colors 1,2,3. Every coloring of $S_{n}$ is obtained by taking a coloring $c_{i}$ of $S_{n-1}$ and choosing a color from $2,3, \ldots, r_{i} \leq k$ for the $n$th vertex, where $r_{i}=\min \left\{k, 1+\right.$ largest color used in $\left.c_{i}\right\}$. Let $c_{i}^{j}$ denote the coloring of $S_{n}$ that agrees with coloring $c_{i}$ on the first $n-1$ vertices and is color $j$ on the $n$th vertex. The following is a Hamilton cycle on all the colorings of $\operatorname{Can}_{k}^{\pi}\left(S_{n}\right)$ :

$$
\begin{gathered}
c_{1}^{4}, c_{1}^{5}, \ldots, c_{1}^{r_{1}}, c_{1}^{3}, c_{1}^{2}, \\
c_{2}^{2}, c_{2}^{4}, c_{2}^{5}, \ldots, c_{2}^{r_{2}}, c_{2}^{3}, \\
c_{3}^{3}, c_{3}^{4}, c_{3}^{5}, \ldots c_{3}^{r_{3}}, c_{3}^{2}, \\
c_{4}^{2}, c_{4}^{4}, c_{4}^{5}, \ldots, c_{4}^{r_{4}}, c_{4}^{3}, \\
\ldots \\
<c_{N}^{2}, c_{N}^{3}>, c_{N}^{5}, \ldots, c_{N}^{r_{N}}, c_{N}^{4}
\end{gathered}
$$

where the order of $<c_{N}^{2}, c_{N}^{3}>$ depends on the parity of $N$.

Part II: Trees with $k>3$ colors. Order the vertices $v_{1}, \ldots v_{n}$ so each vertex $v_{i}$ is a leaf of the tree induced by $v_{1}, \ldots v_{i}$. For $n=4$, the case where $T$ is a star was considered above. The other tree on 4 vertices is a path. A possible ordering of the vertices and the resulting Hamilton cycle for $\operatorname{Can}_{k}^{\pi}\left(P_{4}\right)$ is shown in Figure 2(c). Again we proceed by induction 
on $n$. Let $T^{\prime}=T-v_{n}$. By assumption $\operatorname{Can}_{k}^{\pi}\left(T^{\prime}\right)$ has a Hamilton cycle $c_{1}, c_{2}, \ldots, c_{N}$. We may take $c_{1}$ to be the unique coloring that uses only two colors. For any $i, c_{i}$ can be extended to a coloring of $T$ by coloring $v_{n}$. Let $F_{i}$ be the set of colors for $v_{n}$ compatible with $c_{i}$ and let $\hat{c}_{i}$ be the set of colorings of $T$ that agree with $c_{i}$ on $T^{\prime}$. All colorings of $T$ are obtained this way. Vertex $v_{n}$ can receive any color already used except the one used on its unique neighbor $v_{q}$. If only $r<k$ colors have been used in $c_{i}$ then color $r+1$ can be used as well. Thus $\left|F_{1}\right|=2$ and $\left|F_{i}\right| \geq 3$ for $i>1$.

As in the part I of the proof we will proceed by using all colorings of $\hat{c}_{1}$ followed by those of $\hat{c}_{2}$ etc., returning to $\hat{c}_{1}$. Note that for all $i$ the induced graph on $\hat{c}_{i}$ is complete. Thus we merely need to ensure there is an edge between $\hat{c}_{i-1}$ and $\hat{c}_{i}$ that uses a different vertex in $\hat{c}_{i}$ than some edge between $\hat{c}_{i}$ and $\hat{c}_{i+1}$.

If $c_{i}$ and $c_{i+1}$ use the same color for vertex $v_{q}$ then $\left|F_{i} \cap F_{i+1}\right|=\min \left(\left|F_{i}\right|,\left|F_{i+1}\right|\right)$. If $c_{i}$ and $c_{i+1}$ are different on vertex $v_{q}$ then $\left|F_{i} \cap F_{i+1}\right|=\min \left(\left|F_{i}\right|,\left|F_{i+1}\right|\right)-1$. Thus $\left|F_{i} \cap F_{i+1}\right| \geq 2$ except possibly when $c_{i}, c_{i+1}$ differ on vertex $v_{q}$ and additionally either $i=1$ or $i=N$ (the case $\left|F_{N} \cap F_{1}\right|$ ). Since $c_{1}$ uses just colors 1,2 , the unique coloring that differs from $c_{1}$ only on $v_{q}$ must assign $v_{q}$ color 3 . So either $c_{N}$ or $c_{2}$ must differ from $c_{1}$ on a vertex other than $v_{q}$. Thus at least one of $\left|F_{N} \cap F_{1}\right|$ and $\left|F_{1} \cap F_{2}\right|$ will have 2 elements and we can select an edge from each of $\hat{c_{N}} \hat{c}_{1}$ and ${\hat{c_{1}}}_{1} \hat{c}_{2}$ that use different vertices in $\hat{c}_{1}$.

Part III Trees using exactly 3 colors. A more involved proof is necessary for the case when exactly 3 colors are used to color the tree. The proof is similar to that used by MacGillvray and Choo in [2] to show $\mathcal{C}_{3}(T)$ is Hamiltonian for $T$ not a star.

The proof is by induction on $n=|V(T)|$. Observe that for $n \leq 4, \operatorname{Can}_{3}^{\pi}(T)$ has a Hamilton path. If $T$ is a star, then $\operatorname{Can}_{3}^{\pi}(T)$ has a Hamilton cycle by part I of the proof. Otherwise, consider $T^{\prime}=T-\{u, v\}$ for some pair of leaves $u, v$, that do not have a common neighbor. Such a pair must exist since $T$ is not a star. If $n>4$ the inductive hypothesis gives that $\operatorname{Can}_{3}^{\pi}\left(T^{\prime}\right)$ has a Hamilton path $c_{0}, c_{1}, \ldots, c_{N-1}$.

Order the vertices of $T$ with $u, v$ last and so the order on $T^{\prime}$ is the one that gave the Hamilton path $c_{0}, c_{1}, \ldots, c_{N-1}$. Let $\hat{c}_{i}$ be the induced subgraph of $\operatorname{Can}_{3}^{\pi}(T)$ whose vertex set is the set of canonical 3 -colorings of $T$ that agree with $c_{i}$ on $T^{\prime}$. Clearly, $\hat{c}_{i}$ is a 4 cycle. The Hamilton cycle in $\operatorname{Can}_{3}^{\pi}(T)$ will be constructed so it passes through $\hat{c}_{0}, \hat{c}_{1}, \ldots \hat{c}_{N-2}, \hat{c}_{N-1}, \hat{c}_{N-2}, \ldots \hat{c}_{2}, \hat{c}_{1}, \hat{c}_{0}$, in that order.

Let $u_{0}$ and $v_{0}$ denote the unique vertices adjacent to $u, v \in T$ respectively. Let $\left[\hat{c}_{i}, \hat{c}_{i+1}\right]$ denote the subgraph consisting of the edges between $\hat{c}_{i}$ and $\hat{c}_{i+1}$ in $\operatorname{Can}_{3}^{\pi}(T)$ and the vertices they are incident to. Recall that the colorings $c_{i}$ and $c_{i+1}$ differ on exactly one vertex, say $x$. There are 3 cases.

Case 1: $x \notin\left\{u_{0}, v_{0}\right\}$. Then there is a perfect matching between the 4 vertices in $\hat{c}_{i}$ and $\hat{c}_{i+1}$.

Case 2: $x=u_{0}$. In this case there will be two edges between the vertices in $\hat{c}_{i}$ and $\hat{c}_{i+1}$. We describe the subgraph induced by the vertices in $\hat{c}_{i} \cup \hat{c}_{i+1}$ in this case. Without loss of generality, let the 2 colors available for use on $u$ be $\{1,3\}$ in $\hat{c}_{i}$ and $\{2,3\}$ in $\hat{c}_{i+1}$; and the two colors for use on $v$ be $\{1,2\}$ in both $\hat{c}_{i}$ and $\hat{c}_{i+1}$. The 8 vertices in $\hat{c}_{i} \cup \hat{c}_{i+1}$ are labeled by the colorings on $T^{\prime}\left(c_{i}\right.$ or $\left.c_{i+1}\right)$ followed by the color on $u$ and the color on $v$. Thus the 10 edges will be $\left\{\left(c_{i} 11, c_{i} 12\right),\left(c_{i} 12, c_{i} 32\right),\left(c_{i} 32, c_{i} 31\right),\left(c_{i} 31, c_{i} 11\right)\right\}$ in $\hat{c}_{i},\left\{\left(c_{i+1} 31, c_{i+1} 21\right),\left(c_{i+1} 21, c_{i+1} 22\right),\left(c_{i+1} 22, c_{i+1} 32\right),\left(c_{i+1} 32, c_{i+1} 31\right)\right\}$ in $\hat{c}_{i+1}$, and $\left\{\left(c_{i} 32, c_{i+1} 32\right),\left(c_{i} 31, c_{i+1} 31\right)\right\}$ in $E\left(\left[\hat{c}_{i}, \hat{c}_{i+1}\right]\right)$. 
Case 3: $x=v_{0}$ is similar. Again there are exactly two edges in $E\left(\left[\hat{c}_{i}, \hat{c}_{i+1}\right]\right)$. The vertices they are incident to in $\hat{c}_{i}$ are adjacent, and the vertices in $\hat{c}_{i+1}$ are adjacent.

Next, for each $i$ we construct the two pieces of the cycle $P_{i}, Q_{i}$ that use all vertices of $\hat{c}_{i}$ such that $P_{0} P_{1} P_{2} \ldots P_{N-1} Q_{N-1} \ldots Q_{2} Q_{1}$ is the desired Hamilton cycle.

Step 0 . Construct $P_{0}$. Say the 4-cycle $\hat{c}_{0}$ is $\alpha_{0}, \beta_{0}, \gamma_{0}, \delta_{0}$. Assume without loss of generality that $\alpha_{0}, \delta_{0}$ are adjacent to vertices in $\hat{c}_{1}$, call these vertices in $\hat{c}_{1} \beta_{1}, \gamma_{1}$ respectively. Then define $P_{0}=\alpha_{0}, \beta_{0}, \gamma_{0}, \delta_{0}$.

Step $i . \beta_{i}, \gamma_{i}$ have been defined previously as vertices in $\hat{c}_{i}$ adjacent to vertices in $\hat{c}_{i-1}$. There are several cases. In each case $P_{i}$ will begin with $\gamma_{i}$ and $Q_{i}$ will end with $\beta_{i}$.

Case $u u$. Suppose the colorings $c_{i-1}, c_{i}, c_{i+1}$ differ only by color change on $u_{0}$ (respectively, only on $v_{0}$ ). In this case $u$ (respectively, $v$ ) must take on all 3 colors, so that $V\left(\left[\hat{c}_{i-1}, \hat{c}_{i}\right]\right) \cap V\left(\left[\hat{c}_{i}, \hat{c}_{i+1}\right]\right)=\emptyset$. Label $\alpha_{i}, \delta_{i}$ such that the 4-cycle that is $\hat{c}_{i}$ is $\alpha_{i}, \beta_{i}, \gamma_{i}, \delta_{i}$. Now $\alpha_{i}, \delta_{i}$ are the vertices adjacent to vertices in $\hat{c}_{i+1}$, which we now label $\beta_{i+1}, \gamma_{i+1}$ respectively. Define $P_{i}=\gamma_{i}, \delta_{i}$ and $Q_{i}=\alpha_{i}, \beta_{i}$. Figure 3(Top) gives an example of this case.

Case $u v$. Suppose the colorings $c_{i-1}, c_{i}, c_{i+1}$ differ by color change first on $u_{0}$ and then on $v_{0}$. In this case $V\left(\left[\hat{c}_{i-1}, \hat{c}_{i}\right]\right) \cup V\left(\left[\hat{c}_{i}, \hat{c}_{i+1}\right]\right)$ use only three different vertices of $\hat{c}_{i}$. If $V\left(\left[\hat{c}_{i-1}, \hat{c}_{i}\right]\right) \cap V\left(\left[\hat{c}_{i}, \hat{c}_{i+1}\right]\right)=\gamma_{i}$ then label the rest of the 4 cycle in $\hat{c}_{i}$ so that $\delta_{i}$ is also adjacent to a vertex in $\hat{c}_{i+1}$. Next label the vertices adjacent to $\gamma_{i}, \delta_{i}$ in $\hat{c}_{i+1}$ as $\beta_{i+1}, \gamma_{i+1}$ respectively. Now define $P_{i}=\gamma_{i}$ and $Q_{i}=\delta_{i}, \alpha_{i}, \beta_{i}$. Figure 3(Bottom) gives an example of this case.

Similarly, if $V\left(\left[\hat{c}_{i-1}, \hat{c}_{i}\right]\right) \cap V\left(\left[\hat{c}_{i}, \hat{c}_{i+1}\right]\right)=\beta_{i}$ then label so that $\beta_{i+1}, \gamma_{i+1} \in \hat{c}_{i+1}$ are the vertices adjacent to $\alpha_{i}, \beta_{i}$ respectively. Now define $P_{i}=\gamma_{i}, \delta_{i}, \alpha_{i}$ and $Q_{i}=\beta_{i}$.

Case $u x$. Suppose the colorings $c_{i-1}, c_{i}, c_{i+1}$ differ first by a color change on $u_{0}$ and then on some $x \notin\left\{u_{0}, v_{0}\right\}$. Label $\alpha_{i}, \delta_{i}$ such that the 4-cycle that is $\hat{c}_{i}$ is $\alpha_{i}, \beta_{i}, \gamma_{i}, \delta_{i}$. Now $\alpha_{i}, \delta_{i}$ are adjacent to vertices in $\hat{c}_{i+1}$, which we now label $\beta_{i+1}, \gamma_{i+1}$ respectively. Define $P_{i}=\gamma_{i}, \delta_{i}$ and $Q_{i}=\alpha_{i}, \beta_{i}$.

Case $x x$. Suppose the colorings $c_{i-1}, c_{i}, c_{i+1}$ differ only on vertice(s) not $\left\{u_{0}, v_{0}\right\}$. As in the other cases, label $\alpha_{i}, \delta_{i}$ such that the 4-cycle that is $\hat{c}_{i}$ is $\alpha_{i}, \beta_{i}, \gamma_{i}, \delta_{i}$. Now $\alpha_{i}, \delta_{i}$ are adjacent to vertices in $\hat{c}_{i+1}$, which we now label $\beta_{i+1}, \gamma_{i+1}$ respectively. Define $P_{i}=\gamma_{i}, \delta_{i}$ and $Q_{i}=\alpha_{i}, \beta_{i}$.

Cases $v u, x u, x v$ and $v x$ are similar to those above.

\section{Cycles}

Another simple class of graphs to consider is $C_{n}$, the cycle on $n$ vertices. We prove that there exists an order such that $\operatorname{Can}_{k}^{\pi}\left(C_{n}\right)$ is connected for most $n, k$. While lengthy computer calculations indicate $\operatorname{Can}_{k}^{\pi}\left(C_{n}\right)$ is Hamiltonian for $n>5$, a concise proof is elusive.

Theorem 4.1. For $C_{n}$ a cycle with $n \geq 3$, vertices, and $k \geq 4$ colors, there is an order $\pi$ of the vertices such that $\operatorname{Can}_{k}^{\pi}\left(C_{n}\right)$ is connected. Further, $\operatorname{Can}_{3}^{\pi}\left(C_{4}\right)$ and $\operatorname{Can}_{3}^{\pi}\left(C_{5}\right)$ are connected for some $\pi$ but $\operatorname{Can}_{3}^{\pi}\left(C_{n}\right)$ is disconnected for all $\pi$, for $n>5$.

Proof. The proof is in two parts. 

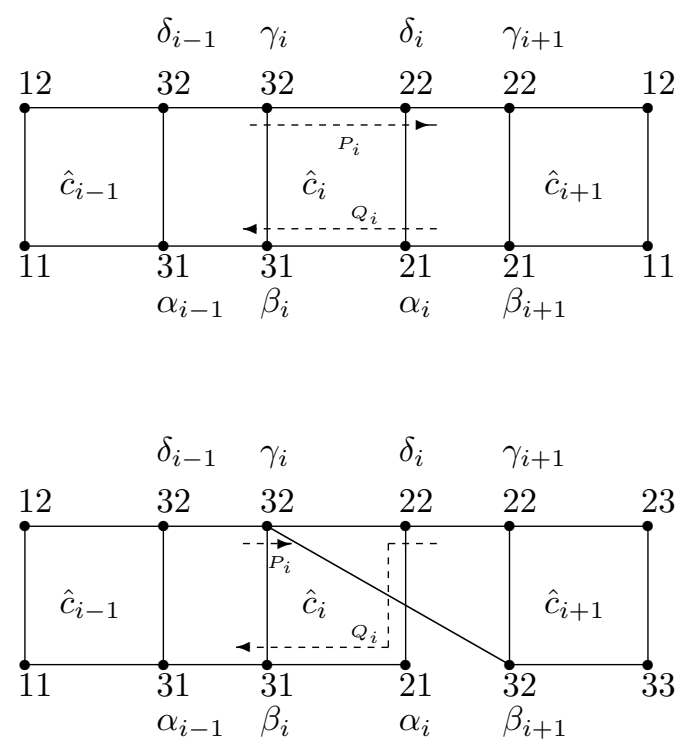

Figure 3: Both figures show possible subgraphs induced by $\hat{c}_{i-1} \cup \hat{c}_{i} \cup \hat{c}_{i+1}$. The numbers (eg 12) give colors on $u, v$ in the underlying graph. The subscripted letters correspond to the notation developed in Step 0 and $i$ of the proof. The dashed lines represent the paths $P_{i}$ and $Q_{i}$. Top: The colorings $c_{i-1}, c_{i}, c_{i+1}$ differ only by color change on $u_{0}$. Bottom: The colorings $c_{i-1}, c_{i}, c_{i+1}$ differ by color change first on $u_{0}$ and then $v_{0}$. 
Part I: $k=3$. It is easy to check that $\operatorname{Can}_{3}^{\pi}\left(C_{4}\right)$ and $\operatorname{Can}_{3}^{\pi}\left(C_{5}\right)$ are connected for many $\pi$ (for example, let the cycle be $(1,2,3,4,5)$ and color in the order given by $\pi=$ $(1,4,2,3,5))$. We show that for $n>5 \operatorname{Can}_{3}^{\pi}\left(C_{n}\right)$ is disconnected. In a 3-colored cycle, the only vertices that can change color are those whose neighbors in the cycle are both the same color. Thus, for $n>5$, when $n=3 t$ the coloring $[1,2,3,1,2,3, \ldots, 1,2,3]$ (where the vertices are listed in the usual order), will be an isolated vertex under any ordering $\pi$. For $n=3 t+2$ consider first $\mathcal{C}_{3}\left(C_{n}\right)$, all colorings of $C_{n}$ in the standard order. Define $L$ to be the set of colorings which for some choice of initial vertex are contiguous substrings of $1,2,3,1,2,3, \ldots 1,2,3$. Colorings in $L$ are only adjacent to other colorings in $L$. For example, consider $[1,2,3,1,2,3, \ldots, 1,2,3,1,2]$ in the usual order. The only colorings this is adjacent to in $\mathcal{C}_{3}\left(C_{n}\right)$, whether canonical or not, are $[3,2,3,1,2,3, \ldots, 1,2,3,1,2]$ and $[1,2,3,1,2,3, \ldots, 1,2,3,1, \mathbf{3}]$, where the bolded number is the changed color. Each of these is also a coloring in $L$, the first by considering the second vertex as the "initial vertex" and the second by considering the last vertex as the initial vertex. Thus any coloring not in $L$ must be in a different connected component of $\mathcal{C}_{3}\left(C_{n}\right)$. Since at least one coloring of $L$ is in $\operatorname{Can}_{3}^{\pi}\left(C_{3 t+2}\right)$ for any ordering $\pi, \operatorname{Can}_{3}^{\pi}\left(C_{3 t+2}\right)$ must also be disconnected.

For $n=3 t+1$ things are slightly more complex. Again consider first the regular coloring graph $\mathcal{C}_{3}\left(C_{n}\right)$. Define $M$ to be the set of colorings consisting two disjoint contiguous substrings of $[1,2,3,1,2,3, \ldots, 1,2,3]$. Colorings in $M$ are only adjacent to other such colorings in $\mathcal{C}_{3}\left(C_{n}\right)$. Without loss of generality suppose the substrings meet as follow $[\ldots, 3,1,2,3,2,3,1, \ldots]$. This is only adjacent to $[\ldots, 3,1,2, \mathbf{1}, 2,3,1, \ldots]$ and $[\ldots, 3,1,2,3, \mathbf{1}, 3,1, \ldots]$ and two more similar colorings at the other end of where the two strings meet. In each case the new coloring simply increases the length of one substring of $[1,2,3,1,2,3,1,2,3, \ldots, 1,2,3]$ and decreases the other. Under any ordering $\pi$ there will be at least one coloring of type $M$ when considered in the usual order, and at least one coloring not of type $M$, so $\operatorname{Can}_{3}^{\pi}\left(C_{3 t+1}\right)$ must also be disconnected.

Part II: $k \geq 4$ colors. For $k \geq 4$ colors, the cases $n=3,4$ are easily verified. For $n \geq 5$, Theorem 3.1 gives a method for finding a Hamilton cycle in $\operatorname{Can}_{k}^{\pi}\left(P_{n-1}\right)$, where $P_{n}$ is the path on $n$ vertices. Let the colorings of $P_{n-1}$ be $c_{1}, c_{2}, \ldots c_{N}$ listed in the order they give a Hamilton cycle and so that $c_{1}$ is the unique coloring that uses only 2 colors.

For any $i, c_{i}$ can be extended to a coloring of $C_{n}$ by coloring $v_{n}$. Let $F_{i}$ be the set of colors for $v_{n}$ compatible with $c_{i}$ and let $\hat{c}_{i}$ be the set of colorings of $C_{n}$. All colorings of $C_{n}$ are obtained this way. Vertex $v_{n}$ can receive any color already used except the ones used on its neighbors $v_{1}$ and $v_{n-1}$. If only $r<k$ colors have been used in $c_{i}$ then color $r+1$ can be used as well. Thus $\left|F_{i}\right| \geq 2$ for $i>1$. Consider first the case where $i \neq 1, N$. If $c_{i}$ and $c_{i+1}$ are the same on vertices $v_{1}, v_{n-1}$ then $\left|F_{i} \cap F_{i+1}\right|=\min \left(\left|F_{i}\right|,\left|F_{i+1}\right|\right) \geq 2$. If $c_{i}$ and $c_{i+1}$ are different on one of the vertices $v_{1}, v_{n-1}$ then $\left|F_{i} \cap F_{i+1}\right|=\min \left(\left|F_{i}\right|,\left|F_{i+1}\right|\right)-1 \geq 1$.

For $i=1$ or $i=N$ consider the cases that $n$ is odd or even separately. If $n-1$ is odd the Hamilton cycle constructed on $\operatorname{Can}_{k}^{\pi}\left(P_{n-1}\right)$ will contain the segment consisting of colorings $c_{N}=[1,2,1,2,1, \ldots, 1,3,1] ; c_{1}=[1,2,1,2,1, \ldots, 1,2,1] ; c_{2}=$ $[1,2,1,2,1, \ldots, 1,2,3]$. Here $\left|F_{N} \cap F_{1}\right|=|\{2,3\}|=2>0$; and $\left|F_{1} \cap F_{2}\right|=|\{2\}|>0$. So there is at least one edge between $\hat{c}_{N}$ and $\hat{c}_{1}$ and also between $\hat{c}_{1}$ and $\hat{c}_{2}$. If $n-1$ is even the Hamilton cycle constructed on $\operatorname{Can}_{k}^{\pi}\left(P_{n-1}\right)$ will contain the the segment consisting of colorings $c_{N}=[1,2,1,2,1, \ldots, 2,1,3] ; c_{1}=[1,2,1,2,1, \ldots, 2,1,2] ; c_{2}=$ $[1,2,1,2,1, \ldots, 2,3,2]$. In this case $F_{N}=\{2,4\} ; F_{1}=\{3\}, F_{2}=\{3,4\}$ so $F_{N} \cap F_{1}=\emptyset$. But since $F_{1} \cap F_{2}=\{3\}$ there is one edge between $\hat{c}_{1}$ and $\hat{c}_{2}$ and $\operatorname{Can}_{k}^{\pi}\left(C_{n}\right)$ is con- 
nected.

Note that the method in this proof is not enough to guarantee a Hamilton Cycle in $\operatorname{Can}_{k}^{\pi}\left(C_{n}\right)$ as it is possible that $\left|F_{i-1} \cap F_{i}\right|=\left|F_{i} \cap F_{i+1}\right|=1$, and each of $\left|F_{i-1}\right|=$ $\left|F_{i}\right|=\left|F_{i+1}\right|=2$. This occurs when each of $c_{i-1}, c_{i}, c_{i+1}$ uses exactly colors $\{1,2,3\}$ and the colors on $\left(v_{1}, v_{n-1}\right)$ are $(1,3),(1,2),(3,2)$ in colorings $c_{i-1}, c_{i}, c_{i+1}$ respectively. In this case $F_{i-1} \cap F_{i}=F_{i} \cap F_{i+1}=\{4\}$, while the possibilities for $v_{n}$ are $F_{i-1}=$ $\{2,4\}, F_{i}=\{3,4\}, F_{i+1}=\{1,4\}$.

It would be interesting to have broad results paralleling Theorems 1.2 and 1.3 for the cannonical coloring graph. Indeed, for all $G$ does there exists $k, \pi$ such that $\operatorname{Can}_{k}^{\pi}(G)$ is connected? In a forthcoming paper the author shows connectivity for some classes of graphs (see [4]).

The author wishes to thank the anonymous referees for their useful suggestions.

\section{References}

[1] L. Cereceda, J. van den Heuvel and M. Johnson, Connectedness of the graph of vertexcolourings, Discrete Math. 308 (2008), 913-919.

[2] K. Choo and G. MacGillivray, Gray code numbers for graphs, Ars Math. Contemp. 4 (2011) 125-139.

[3] M. Dyer, A. Flaxman, A. Frieze and E. Vigoda, Randomly coloring sparse random graphs with fewer colors than the maximum degree, Random Structures Algorithms 29 (2006), 450-465.

[4] R. Haas and G. MacGillivray, Some results on the canonical coloring graph, in preparation.

[5] M. Jerrum, A very simple algorithm for estimating the number of $k$-colorings of a low-degree graph, Random Structures Algorithms 7 (1995), 157-165.

[6] M. Molloy, The Glauber dynamics on colorings of a graph with high girth and maximum degree, SIAM J. Comput. 33 (2004), 721-73.

[7] A. D. Sokal, A personal list of unsolved problems concerning lattice gases and antiferromagnetic Potts models. Inhomogeneous random systems, (Cergy-Pontoise, 2000), Markov Process. Related Fields 7 (2001), 21-38.

[8] E. Vigoda, Improved bounds for sampling colorings, Probabilistic techniques in equilibrium and nonequilibrium statistical physics, J. Math. Phys. 41 (2000), 1555-1569. 